\title{
Emotional faces of children and adults: What changes in their perception
}

\author{
Anna Esposito ${ }^{1,3}$, Antonietta M. Esposito², Gennaro \\ Cordasco $^{1,3}$ \\ ${ }^{1}$ Dipartimento di Psicologia, Università della Campania \\ “Luigi Vanvitelli”, Caserta, Italy \\ ${ }^{2}$ Istituto Nazionale di Geofisica e Vulcanologia, Sez. di \\ Napoli Osservatorio Vesuviano, Italy \\ ${ }^{3}$ International Institute for Advanced Scientific Studies \\ (IIASS), Italy \\ iiass.annaesp@tin.it, antonietta.esposito@ingv.it, \\ gennaro.cordasco@unicampania.it
}

\author{
Mauro Maldonato $^{4}$, Carl Vogel ${ }^{5}$, Nikolaos Bourbakis ${ }^{6}$ \\ ${ }^{4}$ Dipartimento di Neuroscience, Università di Napoli \\ "Federico II", Italy \\ ${ }^{5}$ School of Computer Science and Statistics, Trinity College \\ Dublin, Ireland \\ ${ }^{6}$ Department of Comp. Science \& Eng.,WSU, OH, USA \\ nelsonmauro.maldonato@unina.it; vogel@tcd.ie, \\ nikolaos.bourbakis@wright.edu
}

\begin{abstract}
This work investigates disparities between children and middle aged adults in their ability to decode the six primary facial expressions of emotions when portrayed by contemporary children and adult faces. The analyses were conducted on a sample of 40 (20 females) very closely aged children (mean age $=7.4 ; \mathrm{SD}= \pm 0.2$ ), and 40 middle aged (mean age=54.3; $\mathrm{SD}= \pm 2.9)$ adults (20 females). Four different experimental conditions were assessed: a) 20 children and 20 middle aged adults assessing child faces; b) 20 children and 20 middle aged adults assessing adult faces; c) $\mathbf{2 0}$ children assessing adult faces and 20 children assessing child faces; d) 20 middle aged adults assessing adult faces and $\mathbf{2 0}$ middle aged adults assessing child faces. The analyses do not show significant differences between children and adults for conditions a), and b). Children performances on condition c) did not support the peers-prejudice theory, since no significant differences were found among children on their ability to decode either facial expressions of adults or children. Middle aged adults were significantly more accurate in decoding adult rather than children faces. No significant gender differences were found in the four conditions, even though significant interactions were found between emotional categories and gender of stimuli. In particular, the gender of stimuli had a significant effect in condition a) where emotional faces portrayed by male children are more accurately decoded than those portrayed by female children. Several significant interactions were observed between emotional categories, participants' age, and gender of stimuli. Details are discussed in the text.
\end{abstract}

Keywords - children and adult emotional faces; middle aged adults; emotional decoding accuracy; emotional gender differences; primary emotions

\section{INTRODUCTION}

The correct decoding of emotional facial expressions plays a fundamental role in enabling individuals to successfully communicate and enter into social relationships. The face is a fundamental element in fostering emotional bonds and, from birth, the primary locus of communication and interaction among human beings. The results of a series of developmental studies on the correct decoding of emotional facial expressions have shown that it progresses with age. This is because the necessary skills to allow a correct evaluation and classification of non-verbal information evolve gradually in the course of child's neurological maturation, and their individual cognitive development (Carver et al., 2003; Herba \& Phillips, 2004). During childhood, the recognition of emotional facial expressions is not the same for every single emotion. Durand et al. (2007), in a study involving 100 children aged 5, 7, 9 and 11 years and 26 adults aged between 18-29 years, showed 36 photographs (13 males, 23 females), with vertical or upsidedown faces, each expressing a single emotion among anger, disgust, fear, happiness, sadness, and neutrality. The task was to select an emotional category for each stimulus and define its intensity on a Likert scale from 0 to 10 . The results showed that the recognition of happiness and sadness seems to develop accurately around 5-6 years, with a level of precision very close to that of adults. Fear, on the other hand, does not reach the adult level up to 7 years, anger needs 2 more (9) years, and disgust 4 more (11) years. The authors suggest that the lower accuracy of children than adults in recognizing these emotional categories does not imply that children do not feel these emotions. Rather, their poor ability to recognize them derives from a lack of experience on how these emotions modify facial expressions. Lawrence et al. (2015) investigated the recognition accuracy of the six primary emotional facial expressions on a sample of 478 subjects including children aged 6-11 years and adolescents aged 11-16 years. For this study a computerized version of the Ekman-Friesen Pictures of Facial Affect test (Ekman \& Friesen, 1976) was developed. A total of 60 pictures were presented individually (10 for each emotion) and participants had to click with the mouse on the emotion label (happy, sad, angry, frightened, disgusted and surprised) that best described what they thought was the expressed sentiment. The results showed that accuracy in recognizing facial expressions of happiness, sadness and anger does not differ significantly among children aged between 6 and 16 years. However, the recognition accuracy of fear, disgust and surprise significantly differ among 6 year olds and adolescents. Widen (2013) suggested that children have a systematic way of understanding and interpreting facial 
emotional expressions and other emotional signs. Children start to classify them as "feels good" or "feels bad", and gradually their emotional categories resemble those of adults. The confirmation of the age temporal discrepancy in the recognition of facial emotional expressions comes from another study conducted by Rodger et al. (2015) which demonstrates that the ability to decode such expressions increases from 5 years up to adulthood for all the emotional categories of happiness, fear, disgust and surprise. In contrast, the recognition of facial expressions of sadness and anger remain unchanged from infancy to adulthood.

In a study by Calder et al. (2003) on the recognition of emotional facial expressions in adult life, participants were divided into two groups: 24 subjects between $18-30$ years and 24 aged between 58-70 years. They were shown emotional faces of the six primary emotions and asked to assign them one of the six emotional labels that best described the face stimulus. It was found that, while aging, the ability to recognize facial emotional expressions of fear, anger and sadness decreases significantly compared to the group of youngest participants. These results were confirmed by another research (Isaacowitz et al., 2007) conducted on three groups of participants: young: 18-39 years, middle-aged: 40-59 years, and seniors: $60-85$ years. Subjects were presented emotional faces, in paper mode, and had to select one of the seven emotional labels (happiness, anger, surprise, fear, disgust, sadness and neutral) that best described the stimulus. The authors observed an increased difficulty in recognizing facial emotional expressions of fear and anger with increased age. Furthermore, by comparing the recognition of emotional expressions of the young, middle-aged and senior subjects, the authors find that the last two age groups have comparable performances. The authors concluded that there is less precision in facial emotional recognition skills from middle age. Calder et al. (2003) attribute these results to cognitive deficits due to aging, reporting evidence that the amygdala and hippocampus show neuronal loss and age-related neuronal atrophy through magnetic resonance imaging.

It must be noted that there can be different factors affecting the ability of children and adults to recognize emotional facial expressions, such as for example, the complexity of the task. Bruce et al. (2000) showed that 6 year old children had decoding performances almost similar to adults when they had to indicate between two faces which were happy, sad, angry or surprised. However, when they had to select between two emotional faces expressing the same emotion as a third face, a good level of accuracy was achieved only by 10 year olds (Durand et al., 2007). In addition, gender can play a role. It has been shown that both genders are competent in recognizing a wide variety of emotional facial expressions (Hall \& Matsumoto, 2004). When differences are reported, they typically show a female advantage (Biele \& Grabowska, 2006; Mancini et al., 2013). Another factor influencing the accuracy of the emotional recognition task concerns the characteristics of the presented stimulus. In experiments involving children, stimuli that portray adult faces can result in an underestimation of their ability to correctly decode facial emotional expressions. This is because of an "age-related prejudice" that emerges as the consequence of a social re-orientation towards peers during the developmental period (Scherf et al., 2012; Proietti et al., 2014). It is possible that those same emotional facial expressions, seen on faces of the same age, can be better recognized by peers. Finally, many experimental studies assessing children and adults' ability to recognize facial emotional expressions exploit the Ekman-Friesen Pictures of Facial Affect test, i.e. monochromatic photographs of adults selected during the '70s portraying the six primary emotions of joy, anger, fear, surprise, disgust, and sadness. It is possible that the grayscale photographs of people wearing old-fashioned clothes, makeup and hairstyles, engender different answers compared to more recent and contemporary photographs, producing biased results from differently aged groups.

In light of these considerations, the present work aims to investigate differences in the ability to recognize facial emotional expressions in a specific age group of children, aged between 7.1-7.6 years, and compare their performance with those of middle aged (50-60 years) adults. To this aim, two sets of facial emotional stimuli will be used, representing contemporary emotional facial expressions of the six primary emotions portrayed by both children and adults respectively. The paper aims to investigate at which age and for which gender the recognition is facilitated and whether contemporary children or adult faces are better decoded by peers.

\section{MATERIALS AND PROCEDURES}

The present research focus on the following objectives: 1. Assess differences between children and middle aged adults in their ability to decode the six primary emotional facial expressions portrayed by contemporary faces of children and adults respectively;

2. Assess gender effects both of participants and stimuli.

The research hypotheses are the following:

- Children and/or adults are more skilled in decoding emotional facial expressions of their peers as an outcome of the "age-related prejudice", brain maturation, and brain aging factors (Proietti et al., 2014, Calder et al. 2003, Carver et al., 2003, Herba \& Phillips, 2004);

- There is female advantage in the decoding accuracy of facial emotional expressions (McClure 2000, Biele \& Grabowska, 2006; Mancini et al., 2013);

- Female faces are more accurately decoded than male faces in both groups given that females have been found more emotionally expressive than males (Dimitrovsky et al. 2000, Kret \& De Gelder 2012, McDuff et al. 2017).

\section{A. Material}

The stimuli exploited consisted of 24 adult and 24 child faces portraying the six primary emotional facial expressions of happiness, sadness, anger, fear, surprise, and disgust. Each emotion was represented by two male and two female faces of children and adults, respectively. The stimuli were selected from two larger sets of stimuli, composed of 84 contemporary children faces (14 stimuli for each emotion portrayed by 7 males and 7 females), and 96 contemporary adult faces (16 stimuli for each emotion portrayed by 8 males and 8 females) respectively. Such sets of stimuli were previously assessed by 
two groups, each composed of 40 participants aged between 20-30 years (mean age $=26.22, \mathrm{SD}= \pm 2.87$ ). For the assessment, subjects were presented emotional faces on a computer screen, and had to select one of eight emotional labels (happiness, anger, surprise, fear, disgust, sadness and another emotion, no emotion) that best described the stimulus. In this way, it was possible to assign to each stimulus an accuracy score computed as the percentage of participants' agreement in attributing to the stimulus a given label. The 48 stimuli selected for the present research work were among those that received the highest percentage of label agreements (between 80\% and 100\%), meaning that they were the best accurately decoded.

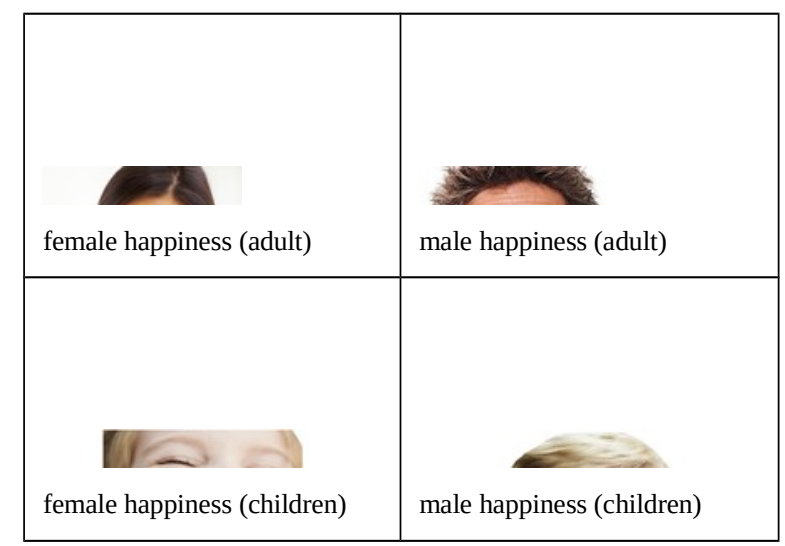

Fig. 1. An example of the proposed stimuli

For the present work, six more stimuli were considered from this database ( 3 of children and 3 of adults) to be exploited in the training phase of each of the two emotional facial recognition tasks proposed to participants. Figure 1 reports examples of the exploited stimuli.

\section{B. Participants}

A total of 80 participants were recruited, 40 children (20 females) aged between 7.1 and 7.6 years (mean age=7.4; $\mathrm{SD}=$ \pm 0.2 ), and 40 adults (20 females) aged between 50-60 years (mean age=54.3; $\mathrm{SD}= \pm 2.9$ ). The recruited children attended the primary school at the Duca D'Aosta Institute in Napoli, Italy. The adults had all obtained a diploma and were recruited among the regular attenders at Santa Maria della Libera oratory in Napoli, and the employees of the Civil Protection Department in Rome, Italy. Each group of 40 was divided in two groups of 20 equally balanced between males and females, which were separately administered the adults and children face tests, respectively. Both adults and children's guardians voluntarily accepted to participate, and signed an informed consent statement. For children, the authorization for conducting the proposed research activity was also given by the Dean of the Duca D'Aosta institute, which acted as representative of the school ethical committee.

\section{Procedure}

Four emotional facial recognition tests were administered, two to adults and two to children, proposing respectively adults and children emotional faces. The visual stimuli were administered individually, through the Superlab software in a quiet room. Each participant saw either only adult or only child emotional facial expressions, presented on a computer screen, and had to select one of the eight labels (happiness, anger, surprise, fear, disgust, sadness, another emotion, and no emotion) that best described the stimulus. No feedback on the accuracy of the response, and no time limits were given.

\section{DATA ANALYSIS}

A series of repeated measure ANOVAs were performed on the correct decoding accuracy obtained by children and adults for the four different conditions under examination: a) Children and middle aged adults assessing child faces; b) Children and middle aged adults assessing adult faces; c) Children assessing adult and child faces; d) Middle aged adults assessing adult and child faces. The ANOVAs were performed on the accuracy responses provided by children and adults. The between factors were the gender (females and males) and age (children and adults) of participants for condition a) and b) and the gender (females and males) and type of stimuli (adult and child faces) for condition c) and d). The within factors were the emotional categories (joy, anger, surprise, fear, disgust and sadness), and gender of the stimuli. The confidence interval was set to alpha $=.05$.

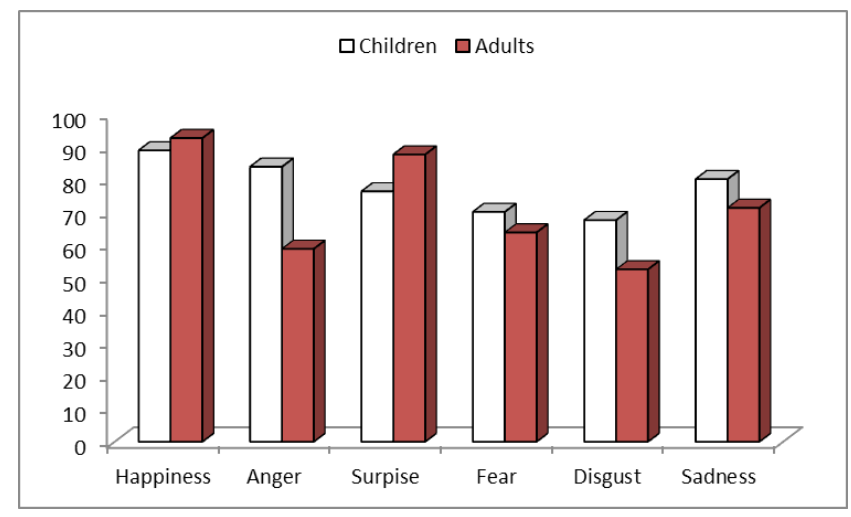

Fig. 2. Children and adults accuracy (\%) on children faces.

\section{A. Chidren and middle aged adults assessing children faces}

No significant differences emerged for age $(F(1,36)=.358$, $\mathrm{p}=.133)$ or gender $(\mathrm{F}(1,36)=.107, \mathrm{p}=.747)$. Children and middle aged adults do not show significant differences in their ability to decode emotional facial expressions of children, even though, children (mean=1.558, $\mathrm{SD}= \pm .06$ ) seem to be more accurate than adults (mean=1.421, $\mathrm{SD}= \pm .06$ ) as illustrated in Fig. 2. Significant differences were found between emotional categories $(\mathrm{F}(5,180)=10.388, \mathrm{p}<<.01)$, suggesting different recognition accuracy depending on the emotion. Bonferroni's post hoc tests show that child happiness is significantly more accurately decoded than anger $(\mathrm{p}<<.01)$, fear $(\mathrm{p}<<.01)$, disgust $(\mathrm{p}<<.01)$ and sadness $(\mathrm{p}=.036)$ by both children and adults.

Significant differences were found with respect to the gender of stimuli $(F(1,36)=7.604, \mathrm{p}<<.01)$. Bonferroni's post hoc tests revealed that stimuli portrayed by male children

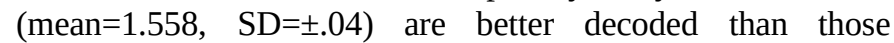

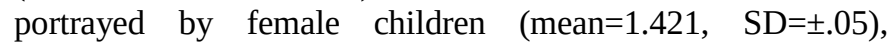
challenging the hypothesis that female faces are more emotionally expressive than male faces. 
A significant interaction was found between emotional categories and gender of stimuli $(\mathrm{F}(5,180)=8.214, \mathrm{p}<<.01)$. Bonferroni's post hoc tests revealed that both children and adults were more accurate in the decoding of male rather than female faces of anger $(\mathrm{p}<<.01)$ and fear $(\mathrm{p}<<.01)$.

A significant interaction was found between groups (children or adults), and emotional categories $(F(5,180)=3.442$, $\mathrm{p}<<.01)$. Bonferroni's post hoc tests revealed that children were more accurate than adults in the recognition of child faces of anger $(\mathrm{p}=.011)$.

\section{B. Children and middle aged adults assessing adult faces}

No significant differences were found between children and adults $(\mathrm{F}(1,36)=1.184, \mathrm{p}=.284)$, and gender $(\mathrm{F}(1,36)=.161$, $\mathrm{p}=.691$ ). Children and middle aged adults recognize emotional facial expressions of adults similarly, even though, children (mean=1.579, $\mathrm{SD}= \pm .05$ ) seem to be less accurate than adults

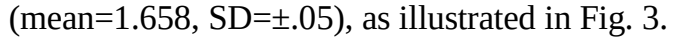

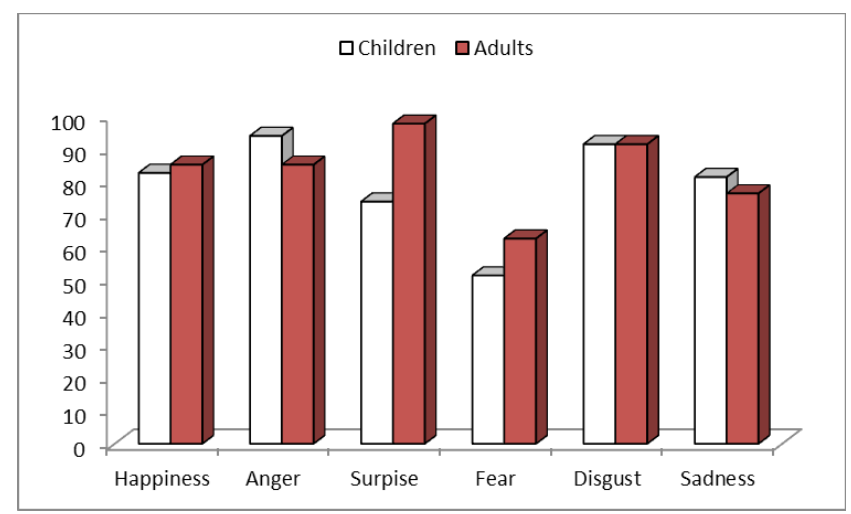

Fig. 3. Children and adults accuracy (\%) on adult faces.

Significant differences were found between the emotional categories $(\mathrm{F}(5,180)=15.022, \mathrm{p}<<.01)$. Bonferroni's post hoc tests revealed that adult fear is significantly less accurately decoded than happiness $(\mathrm{p}<<.01)$, anger $(\mathrm{p}<<.01)$, surprise ( $p<<.01)$, disgust $(\mathrm{p}<<.01)$, and sadness $(\mathrm{p}<<.01)$, by both adults and children.

A significant interaction was found between emotional categories and age group $(\mathrm{F}(5,180)=3.444, \quad \mathrm{p}<<.01)$. Bonferroni's post hoc tests showed that adults were more accurate than children in recognizing the adult faces of surprise $(\mathrm{p}<<.01)$. Analyzing separately children and adult performances, it was found that adult's fear is significantly less well recognized by children than happiness $(p<<.01)$, anger $(\mathrm{p}<<.01)$, disgust $(\mathrm{p}<<.01)$ and sadness $(\mathrm{p}<<.01)$. Adult fear is significantly less well recognized by adults than anger $(\mathrm{p}=.048)$, surprise $(\mathrm{p}<<.01)$ and disgust $(\mathrm{p}<<.01)$, and surprise is significantly better recognized by adults than sadness $(\mathrm{p}<<.01)$.

A significant interaction between emotions and gender of stimuli was found $(F(5,180)=2.550, p=.029)$. Bonferroni's post hoc tests showed that stimuli of happiness portrayed by adult females were significantly more accurately decoded than stimuli of happiness portrayed by adult males $(\mathrm{p}<<.01)$, and stimuli of sadness portrayed by adult males were significantly more accurately decoded than stimuli of sadness portrayed by adult females $(p=.036)$, suggesting that the gender advantage may be contingent on the emotional category.

\section{Children assessing adult and children faces}

No significant differences emerged for the age of stimuli. Children recognize equally well emotional facial expressions portrayed by children and adults $(\mathrm{F}(1,36)=.069, \mathrm{p}=.795)$ as illustrated in Fig. 4. In addition, male and female children have the same performances $(\mathrm{F}(1,36)=.794, \mathrm{p}=.379)$.

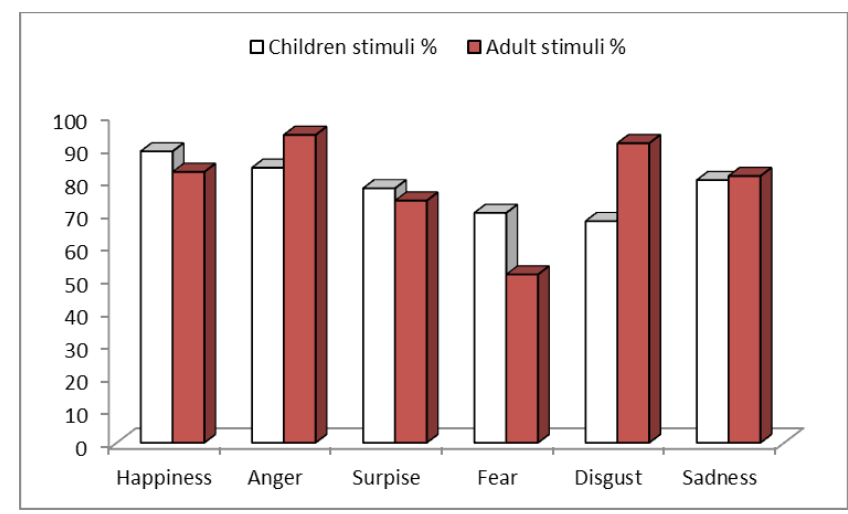

Fig. 4. Children accuracy (\%) on children and adult faces.

Significant differences were found among emotional categories $(\mathrm{F}(5,180)=7.807, \mathrm{p}<<.01)$. Bonferroni's post hoc tests revealed that children decoding of fear is significantly less accurate than happiness $(\mathrm{p}<<.01)$, anger $(\mathrm{p}<<.01)$, disgust $(\mathrm{p}<<.01)$, and sadness $(\mathrm{p}<<.01)$, not matter whether this emotion is portrayed by children or adult faces.

A significant interaction was found between adult and child faces with emotional categories $(\mathrm{F}(5,180)=4.247, \mathrm{p}<<.01)$. Bonferroni's post hoc tests revealed that children are significantly less accurate in decoding disgust in child faces rather than in adult faces $(\mathrm{p}<<.01)$, and fear in adult faces rather than in child faces $(\mathrm{p}=.012)$. Moreover, children who have seen only adult faces were significantly less accurate in decoding adult faces of fear than adult faces of happiness ( $\mathrm{p}<<.01)$, anger $(\mathrm{p}<<.01)$, surprise $(\mathrm{p}<<.01)$, disgust $(\mathrm{p}<<.01)$, and sadness $(\mathrm{p}<<.01)$. On the other hand, children who have seen only children faces, were significantly less accurate in decoding children faces of disgust than children faces of happiness ( $\mathrm{p}=.18$ ) and anger $(\mathrm{p}=.022)$.

A significant interaction was found between adult and child faces with the gender of stimuli $(\mathrm{F}(1,36)=4.610, \mathrm{p}=.039)$. Bonferroni's post hoc tests revealed that female child faces are significantly less accurately decoded than male child faces $(\mathrm{p}=.013)$ challenging again the female advantage hypothesis.

A significant interaction was found between emotional categories and gender of stimuli $(F(5,180)=2.437, p=.036)$. Bonferroni's post hoc tests revealed that children are significantly less accurate in decoding female than male faces of anger $(p=.036)$ and fear $(p=.020)$, suggesting that any gender advantage may be contingent on the emotional category.

Significant interaction was found between emotional categories, gender of stimuli, and age of stimuli 
$\mathrm{F}(5,180)=3.483, \mathrm{p}<<.01)$. Bonferroni's post hoc tests revealed that children are significantly more accurate in decoding fear on male child than male adult faces $(\mathrm{p}<<.01)$, while disgust is significantly better decoded on both male and female adult faces than male $(\mathrm{p}<<.01)$ and female $(\mathrm{p}<<.01)$ child faces.

\section{Adults Assessing Adult and Children Faces;}

A significant difference emerged for the age of stimuli $(\mathrm{F}(1,36)=8.066, \quad \mathrm{p}<<.01)$. Adults are significantly more accurate in decoding adults (mean=1.658) rather than child faces (mean=1.421) as illustrated in Fig. 5. Male and female adults have the same performance $(F(1,36)=2.386, \mathrm{p}=.131)$.

口Children stimuli \% a Adult stimuli \%

Fig. 5. Adult accuracy (\%) on children and adult faces.

Significant differences were found among the emotional categories $(\mathrm{F}(5,180)=13.148, \mathrm{p}<<.01)$. Bonferroni's post hoc tests revealed that adults decoding of happiness (mean=1,775) is significantly more accurate than anger (mean=1.438, $\mathrm{p}=.021$ ), fear (mean $=1.263, \mathrm{p}<<.01$ ), disgust (mean=1.438, $\mathrm{p}<<.01$ ), and sadness (mean=1.475, $\mathrm{p}<<.01$ ). The decoding of surprise (mean=1.850) is significantly more accurate than anger $(\mathrm{p}<<.01)$, fear $(\mathrm{p}<<.01)$, disgust $(\mathrm{p}<<.01)$, and sadness $(\mathrm{p}<<.01)$, while there are no significant differences between happiness and surprise.

A significant interaction was found between the age of stimuli and emotional categories $(\mathrm{F}(5,180)=7.920, \mathrm{p}<<.01)$. Bonferroni's post hoc tests revealed that adults are significantly more accurate in decoding adult faces rather than child faces of anger (child mean $=1.200$, adult mean $=1.700, \mathrm{p}<<.01$ ), surprise (child mean=1.750, adult mean=1.950, $\mathrm{p}=.034$ ) and disgust (child mean=1.050, adult mean=1.825, $\mathrm{p}<<.01$ ). In particular, adults who have seen emotional facial expressions of children are significantly more accurate in decoding child faces of happiness (mean=1.850) rather than child faces of anger (mean=1.175, $\mathrm{p}<<.01$ ), fear (mean=1,275, $\mathrm{p}<<.01$ ), disgust (mean=1.050, $\mathrm{p}<<.01$ ), and sadness (mean=1.425, $\mathrm{p}<<.01$ ). Child facial expressions of surprise (mean=1.750) were decoded by adults significantly more accurately than child facial expressions of anger ( $\mathrm{p}<<.01)$, fear ( $\mathrm{p}<<.01)$, and disgust $(\mathrm{p}<<.01)$. On the other hand, adults who have seen adult faces are significantly less accurate in decoding adult faces of fear (mean=1.250) than adult faces of happiness (mean=1.700, $\mathrm{p}=.02)$, surprise (mean=1.950, $\mathrm{p}<<.01)$ and disgust (mean=1.825, $\mathrm{p}<<.01$ ), as well as, significantly more accurate in decoding adult faces of surprise (mean=1.950) than happiness (mean=1.700, $\mathrm{p}=.02$ ), fear (mean=1.250, $\mathrm{p}<<.01$ ), and sadness (mean=1.525, $\mathrm{p}=.02$ ).

A significant interaction was found between emotional categories and gender of stimuli $(\mathrm{F}(5,180)=3.167, \mathrm{p}<<.01)$. Bonferroni's post hoc tests revealed that male faces of anger are decoded significantly more accurately than female faces of anger $(\mathrm{p}<<.01)$.

A significant interaction was found between age of stimuli, gender of stimuli, and gender of participants $(F(1,36)=4.309$, $\mathrm{p}=.045)$. Bonferroni's post hoc tests revealed that female participants decoded male adult faces significantly more accurately than female adult faces $(p=.046)$. Inversely, male participants decoded female adult faces significantly more accurately than male adult faces $(\mathrm{p}<<.01)$.

\section{DISCUSSION}

This work is an attempt to systematically investigate whether there are differences between children and middle aged adults in their ability to decode the six primary emotional facial expressions when portrayed by contemporary faces of children and adults. An objection made to previous experiments was that they exploited stimuli of non-contemporary faces (as in Lawrence et al. 2015), or that the recognition tasks proposed was made more difficult by requiring implicit cognitive efforts such as vertical or upside-down faces (as in Durand et al. 2007). The choice to compare performances of middle aged adults and children of close age was made considering that both age groups experience changes in thinking, behaving, and feeling. However, while the developmental period is characterized by rapid (from one year to another children change radically) physical, cognitive, and socioemotional changes, during aging similar changes are observed over one or two decades. This justifies the recruitment of children close in the age and middle aged adults which age spans over a decade. According to our first hypotheses, it was expected that children will be more skilled in decoding emotional faces of their peers and the reverse would have been observed for adults as result of the "age-related prejudice" brain maturation, and brain aging factors (Calder et al. 2003, Proietti et al., 2014, Carver et al., 2003, Herba \& Phillips, 2004). This hypothesis was only partially confirmed. Our analyses showed that children recognize equally well children and adults' emotional faces with performances being contingent on emotional categories. Children do not recognize fear on adult faces or disgust in child faces. This is not true for middle-aged adults who are more skilled in decoding adult rather than child faces.

The second hypothesis theorizes a female advantage in the decoding accuracy of facial emotional expressions (McClure 2000, Biele \& Grabowska, 2006; Mancini et al., 2013) no matter whether they are expressed by children or adult faces. This hypothesis was disconfirmed, since no gender differences were observed, neither when recognition accuracy was assessed on children faces, nor on adult faces, or both. These results suggest that both genders, no matter whether they are children or middle aged adults are competent in recognizing a wide variety of emotional facial expressions and the 
differences reported in the literature (McClure 2000, Biele \& Grabowska, 2006; Mancini et al., 2013) should be attributed to other factors such as the complexity of the assigned task, the emotional categories, the gender of participants. This facet deserves to be more investigated.

There is an effect of the gender of stimuli as theorized in the literature (Dimitrovsky et al. 2000, Kret \& De Gelder 2012, McDuff et al. 2017) but not in favor of female faces, since in the condition a) it was observed that stimuli portrayed by male children (mean=1.558, $\mathrm{SD}= \pm .04$ ) are more accurately decoded than those portrayed by female children, and that the gender advantage seems to be contingent on other factors such as emotional categories and participant gender. Also this facet deserves to be more investigated. Additionally, among adults we noted a significant opposite-gender decoding asymmetry: males more accurate at decoding females than males; females more accurate at decoding males than females.

In summary, when the recognition task requires only to assign an emotional label to contemporary emotional faces of either children or adults, emotional categories play the major role on the recognition accuracy of both children and middle aged adults. Emotional categories are shown to interact significantly with other factors such as age of participants, age of stimuli, and gender of stimuli, suggesting that the six primary emotions weigh differently on the human perceptual ability to recognize them as a function of contextual instances. These facets deserve to be more investigated when the implementation of emotionally and socially behaving user interfaces is on the stage.

\section{CONCLUSION}

This work, as suggested by one of the reviewers, focus on purely natural cognitive capabilities, i.e. the human ability to decode emotional faces. However, the hope is that the reported data provide the basic knowledge needed for a better modeling of artificial cognitive capabilities such as the automatic recognition of faces on mobile platforms, automatic detection of empathic actions/reactions, and synthesis of sympathetic or annoyed faces as ICT-AI (Information Communication Technologies and Artificial Intelligence) solutions to improve the quality of life of end users and support vulnerable people in their daily routines (Bertok \& Fazekas 2016, Alam et al. 2016, Irastorza \& Torres 2016, Lewandowska-Tomaszczyk and Wilson, 2016).

\section{ACKNOWLEDGMENTS}

The research leading to the results presented in this paper has been conducted in the project EMPATHIC (grant n. 769872) that received funding from the European Union's Horizon 2020 research and innovation programme.

\section{REFERENCES}

[1] Alam, F., Danieli, M., Riccardi, G. (2016) Can we detect speakers' empathy? A real-life case study. In Proc. of IEEE 7th CogInfoCom2016, Wroclaw, Poland, October 16-18.

[2] Biele, C., Grabowska, A. (2006) Sex differences in perception of emotion intensity in dynamic and static facial expressions. Experimental Brain Research. 171(1), 1-6
[3] Bruce, V., Campbell, R.N. Doherty-Sneddon G., Import A., Langton S.,McAuleyS., Wright R. (2000) Testing face processing skills in children. British Journal of Developmental Psychology, 18,319-333.

[4] Calder, A.J., Keane, J., Manly,T., Sprengelmeyer, R., Scott, S., NimmoSmith, I., Young, A.W. (2003). Facial expression recognition across the adult life span. Neuropsychologia, 4, 195-202.

[5] Carver, L.J., Dawson, G., Panagiotides, H., Meltzoff, A.N., McPartland, J., Gray, J., Munson, J. (2003) Age-related differences in neural correlates of face recognition during the toddler and preschool years. Developmental Psychobiology, 42(2), 148-159.

[6] Dimitrovsky, L., Spector, H., Levy-Shiff, R. (2000) Stimulus gender and emotional difficulty level. Their effect on recognition of facial expressions of affect in children with and without LD. Journal of Learning Disabilities, 33(5), 410-416.

[7] Durand, K., Gallay, M., Seigneuric, A., Robichon, F., Baudouin, J.Y. (2007). The development of facial recognition: The role of configural information. Journal of Experimental Child Psychology, 14-27

[8] Ekman, P., Friesen, W.V. (1976) Measuring facial movement. Environmental psychology and nonverbal behavior. 1(1), 56-75.

[9] Hall, J.A., Matsumoto, D. (2004). Gender differences in judgments of multiple emotions from facial expressions. Brief Report, 4(2),201-206.

[10] Herba, C., Phillips, M. (2004) Development of facial expression recognition from childhood to adolescence: Behavioural and neurological perspectives. Journal of Child Psychology and Psychiatry, 45(7), 1185-1198.

[11] Irastorza, J, Torres, M.I. (2016) Analyzing the expression of annoyance during phone calls to complaint services. In Proc. of IEEE 7th CogInfoCom2016, Wroclaw, Poland, October 16-18.

[12] Isaacowitz, D.M., Löckenhoff, C.E., Lane, R.D., Wright, R., Sechrest, L., Riedel, R., Costa, P.T. (2007) Age differences in recognition of emotion in lexical stimuli and facial expressions. Psychology and Aging. 22(1), 147-159.

[13] Kret, M.E., De Gelder, B.A. (2012) Rreview on sex differences in processing emotional signals. Neuropsychologia, 50(7), 1211-1221.

[14] Bertok, K., Fazekas, A. (2016) Face recognition on mobile platforms. In Proc. of IEEE 7th CogInfoCom2016, Wroclaw, Poland, October 16-18.

[15] Lawrence., K., Campbell, R., Skuse, D. (2015). Age gender and puberty influence: The development of facial emotion recognition. Front Psychol. 16, 6:761.

[16] Lewandowska-Tomaszczyk, B., Wilson, P. (2016) Compassion, empathy and sympathy expression features in affective robotics. In Proc. of IEEE 7th CogInfoCom2016, Wroclaw, Poland, October 16-18.

[17] Mancini, G., Agnoli, S., Baldaro, B., Ricci Bitti, P.E., Surcinelli, P., (2013) Facial expressions of emotions: Recognition accuracy and affective reactions during late childhood. The Journal of Psychology, 147(6), 599-617.

[18] McDuff, D., Kodra, E., Kaliouby, R., LaFrance, M. (2017) A large-scale analysis of sex differences in facial expressions. PLoS One. $2017 \mathrm{Apr}$ 19;12(4):e0173942. doi: 10.1371/journal.pone.0173942

[19] McClure, E.B. (2000) A meta-analytic review of sex differences in facial expression processing and their development in infants, children and adolescents. Psychological Bulletin, 126(3), 424-453.

[20] Proietti, V., Macchi Cassia, V., Mondloch, C.J.(2014) The own-age face recognition bias is task dependent. British Journal of Psychology, 106(3), 446-467.

[21] Rodger, H.,Vizioli, L., Ouyang, X., Caldara, R. (2015) Mapping the development of facial expression recognition. Developmental Science, 18(6), 926-939.

[22] Scherf, K.S., Behrmann, M., Dahl R.E. (2012) Facing changes and changing faces in adolescence: A new model for investigating adolescent-specific interactions between pubertal, brain and behavioral development. Developmental Cognitive Neuroscience, 2(2), 199-219.

[23] Widen, S.C. (2013) Children's interpretation of facial expressions: The long path from valence-based to specific discrete categories. Emotion Review.5(1),72-77 
